$\Sigma_{n=1}^{N}\left(j_{n}(N)-j_{n}\right)^{2}$ goes to zero as $N$ approaches infinity, where $\left\{j_{n}\right\}$ is the solution to equation (2).

These last two theorems are applied in considering the potential problem involving the temperature in a sphere having prescribed temperature in the top half and Newtonian heat loss through the lower half. (In fact, a survey of some seventy papers involving dual orthogonal series shows that these last two theorems are sufficiently general to apply to all of them.)

\title{
REFERENCES
}

1. R. P. Feinerman and R. B. Kelman, The convergence of least square approximations for dual orthogonal series, Glasgow Math. J. 15 (1974), 82-85.

2. R. B. Kelman and R. P. Feinerman, Dual orthogonal series, SIAM J. Math. Anal. 5 (1974), 489-502.

3. I. N. Sneddon, Mixed boundary value problems in potential theory, NorthHolland, Amsterdam; Interscience, New York, 1966. MR 35 \#6853.

DEPARTMENT OF MATHEMATICS, LEHMAN COLLEGE, CITY UNIVERSITY OF NEW YORK, BRONX, NEW YORK 10468

DEPARTMENT OF COMPUTER SCIENCE, COLORADO STATE UNIVERSITY, FORT COLLINS, COLORADO 80523

BULLETIN OF THE

AMERICAN MATHEMATICAL SOCIETY

Volume 81, Number 4, July 1975

\section{CONFORMAL GEOMETRY IN HIGHER DIMENSIONS. I}

\author{
BY R. S. KULKARNI
}

Communicated ${ }^{1}$ February 24, 1975

Conformally Euclidean manifolds are one type of higher dimensional generalization of Riemann surfaces. They are studied and classified here from that point of view (cf. [2] and [3]).

1. Definition 1.1. A conformal structure on a manifold $M$ is a covering $\left\{U_{\alpha}\right\}$ together with a metric $g_{\alpha}$ on $U_{\alpha}$ such that whenever $U_{\alpha} \cap U_{\beta} \neq \varnothing, g_{\alpha}$ and $g_{\beta}$ are conformally related on $U_{\alpha} \cap U_{\beta}$.

AMS (MOS) subject classifications (1970). Primary 53A30.

1 Originally received July 24, 1974. 
An advantage of this notion is that a conformally Euclidean manifold has an analytic conformal structure. Moreover

THEOREM 1.2. A conformally Euclidean manifold admits an analytic Riemann metric compatible with the conformal structure.

CoRollary 1.3. A connected sum of two copies of $S^{n-1} \times S^{1}$ carries an analytic conformally Euclidean metric which cannot be realized in $\mathbf{R}^{n+1}$ if $n \geqslant 4$ although it carries $C^{\infty}$ conformally Euclidean metrics which are realizable in $\mathbf{R}^{n+1}$ (cf. [3]).

2. The model space and the canonical bundle. The group $\mathfrak{M}_{n}$ of conformal automorphisms of the standard $n$-sphere is a Lie group isomorphic to two out of the four components of $O(n+1,1) .\left(S^{n}, \mathfrak{M}_{n}\right)$ is the model space for $n$ dimensional conformal geometry. Assume $n \geqslant 3$. A basic theorem of Liouville says that a conformal map $U \rightarrow S^{n}$, $U$ open nonempty in $S^{n}$ is the restriction of a unique element of $\mathfrak{M}_{n}$. It follows that a 1-cocycle arising from a conformal structure on a conformally Euclidean $M^{n}$ defines an $S^{n}$-bundle $\xi$ on $M^{n}$ with structure group $\mathfrak{M}_{n}$ and a canonical section $\sigma$ which in a natural sense is transversal to the base. This bundle is implicit in Kuiper [2] and in much of the work on Kleinian groups (cf. also Gunning [1]). The induced homomorphism $\rho: \pi_{1}(M) \rightarrow \mathfrak{M}_{n}$ is called the conformal holonomy representation. If $k=\operatorname{ker} \rho$ and $M_{k}$ is the corresponding covering space then $\xi$ pulled back to $M_{k}$ is trivial and the corresponding section $\sigma_{k}$ gives rise to the development map $\delta: M_{k} \rightarrow S^{n}$. We call $\Omega_{M}=\delta\left(M_{k}\right)$ the planar model of $M$. $M$ is called Kleinian (resp. weakly Kleinian) if im $\rho$ is discontinuous on $\Omega_{M}$ (resp. discrete in $\mathfrak{M}_{n}$ ).

3. Class and kind. Write $\Omega$ for $\Omega_{M}$. Let $\partial \Omega$ be its set theoretic boundary. If $M$ is Kleinian and compact $\alpha \Omega$ coincides with the closure of the set of fixed points of nonelliptic elements of im $\rho$. The notation $S^{p}$ will denote the standard $p$-sphere if $p \geqslant 0$, a point if $p=-1$, and the empty set if $p=-2$.

DEFINITION 3.1. $M$ is said to be of class $p,-2 \leqslant p \leqslant n$, if $\partial \Omega$ is contained in some $S^{p} \subseteq S^{n}$ but not in any $S^{p-1} \subseteq S^{n}$. Moreover it is said to be of the first (resp. second) kind if $\partial \Omega=S^{p}$ (resp. $\left.\partial \Omega \neq S^{p}\right)$.

Of course if $p \leqslant 0$ there is no "second kind" and if $p=n$ there is no "first kind." It is seen that $p=-2$ (resp. -1 , resp. $n-1$ ) and first kind corresponds to a spherical (resp. Euclidean, resp. hyperbolic) space form.

THEOREM 3.1. There exist compact manifolds of all classes and kinds. 
4. A conformal surgery. In [3] it was announced that a connected sum of conformally Euclidean manifolds carries a conformally Euclidean structure. We take this opportunity to point out that Professor Milnor pointed out to us that Theorem 1 in [3] is not correct as stated. Since then we have come to realize a close connection of this construction with the "combination theorems" of Klein and Maskit in the theory of Kleinian groups. A general principle may be extracted as follows: Let $M^{n}$ and $N^{n}$ be manifolds with homeomorphic boundaries $\partial M \approx^{\varphi} \partial N$. Let $M \#_{\varphi} N$ denote the manifold obtained by glueing $M$ with $N$ along the boundaries by $\varphi$. Let $\partial M$ and $\partial N$ have collar neighborhoods $U=\partial M \times[-\epsilon, \epsilon], V=\partial N \times[-\epsilon, \epsilon]$ where $\partial M$ (resp. $\partial N$ ) is identified with $\partial M \times\{-\epsilon\}$ (resp. $\partial N \times\{-\epsilon\})$. If there exists a conformal map $H: U \rightarrow V$ such that $H(x, t)=\left(f_{t}(x),-t\right), x \in \partial M, t \in[-\epsilon, \epsilon]$, then identifying $U$ with $V$ via $H$ gives a conformally Euclidean structure on $M \#_{\varphi} N$. This structure depends on the map $H$ which reflects on the deformations of a conformal structure on $M \#_{\varphi} N$. An example of such a map $H$ is the inversion in the equatorial $S^{n-1} \subset S^{n}$. We formulate a general

DEFINITION 4.1. Let $M^{n-1}$ be a compact smooth submanifold of $S^{n}$. An inversion in $M^{n-1}$ is a differentiable involution $\alpha: S^{n} \rightarrow S^{n}$ which leaves $M^{n-1}$ invariant so that the induced map on the normal bundle of $M^{n-1}$ is orientation-reversing. We shall call $M^{n-1}$ a trace of the inversion $\alpha$.

THEOREM 4.2. Given $g=0,1,2, \ldots$ : There exist conformal inversions of $S^{3}$ whose trace is a compact surface of genus $g$.

It is also possible to describe such inversions in higher dimensions. These are possible candidates for the map $H$ above and show the possibility of higher dimensional analogues of the Klein Maskit combination theorems.

\section{REFERENCES}

1. R. C. Gunning, Special coordinate coverings of Riemann surfaces, Math. Ann 170 (1967), 67-86. MR 34 \#7790.

2. N. H. Kuiper, On conformally-flat spaces in the large, Ann. of Math. (2) 50 (1949), 916-924 and 51 (1950), 478-490. MR 11, 133.

3. R. S. Kulkarni, Conformally flat manifolds, Proc. Nat. Acad. Sci. USA 69 (1972), 2675-2676.

DEPARTMENT OF MATHEMATICS, COLUMBIA UNIVERSITY, NEW YORK, NEW YORK 10027 